\section{Child Findings from a 10-Week Multi- Component Family Meals Intervention Targeting Underserved Families with Elementary Aged Children}

Catherine Rogers, MS, LDN, RD, The Ohio State University; Carolyn Gunther, PhD, gunther.22@osu.edu, 1787 Neil

Avenue, Columbus, $\mathrm{OH} 43210$

Objective: Assess the effectiveness of a 10-week multicomponent family meals intervention, Simple Suppers (SS), aimed at eliciting positive changes in child diet and weight.

Study Design, Setting, Participants, Intervention: Simple Suppers was implemented over 12-months as a two-group (intervention, waitlist control) quasi-experimental trial using a staggered cohort design. The program targeted underserved, racially diverse families with children 4-10 year olds SS included 10, 90-minute lessons delivered weekly over the dinner hour at faith-based community center.

Outcome Measures and Analysis: Child outcomes included change in body mass index z-score (zBMI), daily serving of fruits, vegetables, and sugar-sweetened beverages (SSBs), assessed at baseline, post-intervention, and 10-week follow-up. Generalized Linear Mixed Models (GLMM) were used to compare response variable results of intervention to control.

Results: One hundred and twenty-six children completed the program (intervention $=86$; control $=40$ ). Mean age was 6.9 years old, $62 \%$ were female, and $60 \%$ were Black. Mean baseline zBMI was 0.69. There were no significant differences in zBMI between groups from pre- to post-intervention. However, among intervention children, there was a significant difference in zBMI by level of attendance with high ( $>7$ lessons) vs low $(<7)$ attenders having a lower zBMI from pre- to post-intervention $(\mathrm{p}=0.017)$. $\mathrm{zBMI}$ was maintained among intervention children at follow-up $(p=0.493)$. There were no significant differences in fruits, vegetables, or SSBs between groups from pre- to post-intervention, nor was an attendance effect observed. A significant increase in vegetables was observed among intervention children at follow-up $(\mathrm{p}<0.001)$.

Conclusions and Implications: Participation in Simple Suppers at a 70\% level led to decreased zBMI, but no immediate improvements in child dietary outcomes. Results from this study demonstrate the potential for engagement in family meals program to positively impact child weight among racially diverse children.

Funding: Cardinal Health Foundation
054 The Childhood Health, Education, \& Wellness (CHEW) Program: Lessons Learned from a Weight Management Program Targeting Childhood Obesity Among Latino Children and their Families

Sara Kohn Rhoades, MS, srhoades@masonlive.gmu.edu, George Mason University, 4400 University Drive MSN 1F8, Fairfax, VA 22030; Julizza Canales, George Mason University; Kathy Sanchez, MS, CSN; Sina Gallo, PhD, RD

Objective: Design and pilot-test a culturally acceptable, family-centered weight management program for overweight to obese Latino children.

Target Audience: Latino children with a BMI-for-age $>85$ th percentile (CDC), age 6-11 years, and their parents. Children were recruited through local schools; all were from low-income families.

Theory, Prior Research, Rationale: There is a dearth of studies focused on individualized nutrition counseling with Latino children; most are short-term group sessions and do not address home environment, cultural perceptions, or food children consume outside school. The program was informed by social cognitive theory (SCT) to understand behavioral and environmental factors influencing child/family eating behavior. The transtheoretical model provided the basis for integrating SCT constructs to assess behavior change.

Description: Children and their families $(n=12)$ were randomized to immediately begin individualized monthly counseling sessions with a bilingual nutrition educator or were wait-listed controls. The 5-month program focused on both nutrition and activity behaviors and included five 1-hour lessons adapted to the Latino culture and delivered in Spanish: MyPlate; portion sizes; food labels; meal plans; eating out. Parent-child dyads set SMART goals at each session and self-monitored food intake and activity using 3-day food records and logs.

Evaluation: Retention rate at follow-up was 83\%. Subgroup results $(n=5)$ indicate that all families felt more confident in making healthier food choices and parents observed positive changes in their child's eating and physical activity behaviors.

Conclusions and Implications: An individualized family-based nutrition counseling approach improved self-efficacy among children and parents. Further studies evaluating program effectiveness at a larger scale are warranted.

Funding: Academy of Nutrition and Dietetics, Ann A. Hertzler Grant 